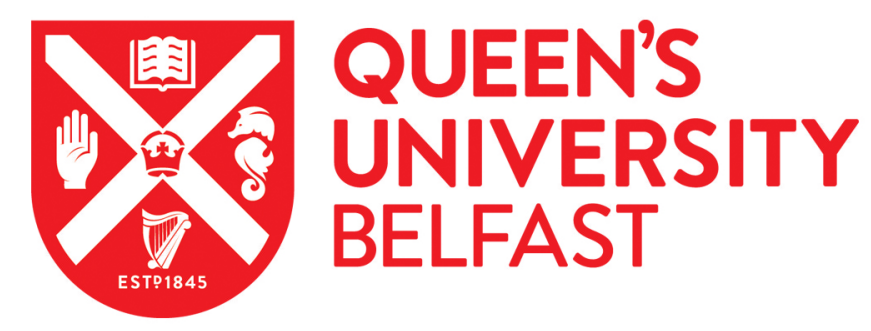

\title{
Gaussian Process Multi-Class Classification for Transformer Fault Diagnosis using Dissolved Gas Analysis
}

Wang, L., Littler, T., \& Liu, X. A. (2021). Gaussian Process Multi-Class Classification for Transformer Fault Diagnosis using Dissolved Gas Analysis. IEEE Transactions on Dielectrics and Electrical Insulation, 28(5), 1703 - 1712. https://doi.org/10.1109/TDEl.2021.009470

\section{Published in:}

IEEE Transactions on Dielectrics and Electrical Insulation

\section{Document Version:}

Peer reviewed version

Queen's University Belfast - Research Portal:

Link to publication record in Queen's University Belfast Research Portal

\section{Publisher rights}

Copyright 2021, IEEE.

This work is made available online in accordance with the publisher's policies. Please refer to any applicable terms of use of the publisher.

\section{General rights}

Copyright for the publications made accessible via the Queen's University Belfast Research Portal is retained by the author(s) and / or other copyright owners and it is a condition of accessing these publications that users recognise and abide by the legal requirements associated with these rights.

Take down policy

The Research Portal is Queen's institutional repository that provides access to Queen's research output. Every effort has been made to ensure that content in the Research Portal does not infringe any person's rights, or applicable UK laws. If you discover content in the Research Portal that you believe breaches copyright or violates any law, please contact openaccess@qub.ac.uk. 


\title{
Gaussian Process Multi-Class Classification for Transformer Fault Diagnosis using Dissolved Gas Analysis
}

\author{
Lin Wang, Tim Littler and Xueqin Liu \\ School of Electronics, Electrical Engineering and Computer Science \\ Queen's University Belfast \\ Belfast, BT9 5AH, UK
}

\begin{abstract}
Dissolved gas analysis (DGA) is widely used for oil-immersed power transformers as a conventional fault diagnosis tool. However, interpretation criteria from DGA assessment often depends on empirical discrimination from a specialist, which can render unreliable or ambiguous diagnoses. Intelligent fault classification algorithms can be implemented to conquer uncertainty in conventional methods, and which require feature learning of transformer condition information data rather than expert experience. In this paper, a Gaussian process multi-classification (GPMC) method is proposed, which uses multiclass recognition with a Gaussian process (GP) and renders an output with a probabilistic interpretation rather than a deterministic guess. The method is investigated using largescale DGA field datasets to improve diagnostic accuracy, and presents reliable incipient fault diagnosis ability. A kernel-based learning algorithm and versatile artificial intelligence (AI) methods, (support vector machine (SVM), artificial neural network (ANN), K-nearest neighbors (KNN), decision tree and logistic regression (LR)) have been used to obtain comparative classification accuracy in comparison to the proposed method: additional comparison is demonstrated between conventional DGA and AI methods. The effectiveness and robustness of the proposed GPMC method are confirmed by experimental accuracy $>95 \%$, which illustrates that the proposed method is able to provide superior and reliable diagnoses for operational transformer faults.
\end{abstract}

Index Terms - transformer fault diagnosis, DGA, Gaussian process multiclassification, support vector machine, artificial neural network, $k$-nearest neighbors, decision tree, logistic regression, conventional DGA methods.

\section{INTRODUCTION}

POWER transformers are critical and high capital investment assets in electric power systems which require regular assessment for maintenance management. Awareness of transformer health requires condition monitoring to limit outage of service, expensive downtime and excess maintenance costs [1].

High voltage oil-immersed transformers - for both transmission $(>100 \mathrm{kV})$ and distribution $(<100 \mathrm{kV})$ systems - are typically subject to internal faults which occur by decomposition of insulation from electrical and thermal stresses. Decomposition of oil insulation generates a quantity of hydrocarbon dissolved in oil: carbon oxides emerge with the deterioration of insulating paper. Ethylene $\left(\mathrm{C}_{2} \mathrm{H}_{4}\right)$, ethane $\left(\mathrm{C}_{2} \mathrm{H}_{6}\right)$, carbon dioxide $\left(\mathrm{CO}_{2}\right)$, carbon monoxide $(\mathrm{CO})$ are rendered by thermal influences; hydrogen $\left(\mathrm{H}_{2}\right)$, methane $\left(\mathrm{CH}_{4}\right)$ and acetylene $\left(\mathrm{C}_{2} \mathrm{H}_{2}\right)$ are caused by electrical faults including partial discharge and arcing [2]. Dissolved gas analysis (DGA) is employed extensively in assessment of transformer faults and recognition. Several conventional methods [3] are also applied to interpret DGA outcomes, such as the key gas

Corresponding author: L. Wang

E-mail:lwang15@qub.ac.uk method, Rogers ratio method, Dornenburg ratio method, IEC ratio method and Duval triangle method. However, these methods generally depend on subjective expertise, based on accumulated experience: diagnostic results may therefore vary - even for the same sample data, resulting in potential conflict and uncertainty among different interpretation approaches. In recent years, with the purpose of realizing more precise and reliable prediction, artificial intelligence (AI) approaches and algorithms have been developed in the general fault diagnosis area.

In [4], the adaptive network-based fuzzy inference system (ANFIS) aggregated with the Dempster-Shafer theory (DT) has been presented to improve fault diagnosis capabilities, based on DGA which helps eliminate the existence of incorrect or unresolved diagnoses. A joint model of fuzzy association rules and case-based reasoning (CBR) was implemented for transformer diagnosis by connecting fault type with characteristic parameters to enhance diagnostic capability [5]. The performance of rulebased fuzzy logic approaches relies on the empirical and cumulative knowledge of human experts, thus, some prevalent AI models extract relative state information from training data directly rather than manual collection, such as artificial neural network (ANN) and support vector machines (SVMs).

Graphical approaches have been used for fault recognition 
combined with AI methods: in [6], the Duval method was combined with SVM and k-nearest neighbors (KNN); the Triangle method and Pentagon method were used to realize fault diagnosis in [7]; in [8], feature selection methods (wrapper, information gain, relief and correlation-based feature selection) were integrated with different classification algorithms (decision tree, SVM, ANN and $\mathrm{KNN}$ ) to define transformer health indices. To understand the change between different health states in a transformer, a Hidden Markov Model (HMM) was proposed to obtain transition probability in [9]. A Bayesian network combined hypothesis testing was proposed in [10] to assess transformer faults based on probabilistic outputs: $88.9 \%$ accuracy was validated with the IEC TC10 dataset. To develop a distinctive relationship between dissolved gas and specific fault types, a combination of multinomial logistic regression model and back-propagation neural network (BPN) was proposed in [11]. A deep belief network (DBN), which acquires detailed features from data samples, achieves an effective fault diagnosis through a layer-by-layer structure in [12]. However, one of the main shortcomings of a network approach is the "black-box" structure, which limits the interpretability of internal interrelations and operational processes.

This paper proposes a method based on a computational model to provide a clear discrimination between a transformer normal (healthy) state and incipient fault conditions, thereby supplying accurate diagnostics to preempt maintenance and avoid or reduce potential breakdown. The main contributions of this paper are: 1) propose a Bayesian based Gaussian approach - Gaussian process multi-classification (GPMC) to eliminate uncertainty and render probabilistic results to clearly identify fault location rather than ambiguous estimation; 2) use a hybrid approach to process unlabeled DGA datasets (datasets in this paper have been supplied by General Electric) to build a database with labelling, using a combination of Rogers Ratio and the Duval Triangle method (the purpose of the database to assist AI models for classification of otherwise unknown datasets - details can be seen in [13]); 3) distinguish between normal state and specific fault classes using large-scale DGA field data (comprising 7000 sample points: 5000 fault datasets +2000 normal datasets) to confirm reliability in the proposed method (the conventional models-SVM, ANN, KNN, decision tree and logistic regression (LR) have been used for comparative assessment of classification accuracy); and 4) establish a comparison of conventional DGA interpretation - using the Dornenburg ratio method, IEC 60599 and key gas methods with intelligent methods to explore the capacity of AI models for transformer fault diagnosis.

Gaussian processes are mathematically equivalent or related to many well-known models. Existing work has demonstrated that large neural networks with infinite hidden layers are equivalent to Gaussian Process with certain covariance function: further detail may be found in [14], [15]. A Gaussian process is attractive for small data sets as a non-parametric Bayesian model. In comparison, neural networks as parametric models are difficult to train in practice due to difficulties in selecting suitably large parameters, architecture, and activation functions. Therefore, the GPMC approach was proposed for the transformer condition classification, which is also particularly beneficial for less common and infrequent fault types.

This paper is structured as follows. Section 2 introduces the implementation approach of the GPMC model used in this work. Section 3 presents the AI methods used in this paper. Section 4 compares the performance of GPMC with established models, discusses results from a chosen case study, and verifies the robustness of training data and test data split. Section 5 summarizes paper contributions and identifies future work.

\section{GAUSSION PROCESS FOR CLASSIFICATION}

\subsection{GAUSSIAN PROCESS BINARY CLASSIFICATION}

Kernel methods have been widely investigated in machine learning for data feature extraction, applied effectively in SVM and the Gaussian process classifier (GPC). A Gaussian process (GP) can be described as stochastic for a combination of random variables. William et al. [16] applied a GP to Bayesian inference to represent prior distribution for data regression. A Gaussian process classifier (GPC) subject to non-parametric probability classification, implemented based on Bayesian inference for priors, has been discussed in [17]. The classification model calculates the probability $p(y, x)$, where $y$ indicates the class label represented by \pm 1 for binary classification. Figure 1 presents the structure of a GPC: a latent function $f$ is introduced to map the input value into unit interval; the probability distribution of latent function is used for computing the class probability, $\pi\left(x_{*}\right)=p\left(y_{*}=1 \mid f_{*}\right)$. Given the set of data points: $\mathrm{D}=(X, y)=\left\{\left(x_{i}, y_{i}\right) \mid i=1,2, \ldots, \mathrm{n}\right\}, X=$ $\left\{x_{i} \mid i=1,2, \ldots, \mathrm{n}\right\}$, then the target label $y_{i} \in\{-1,1\}$, and $n$ denotes the number of sample data. A sigmoidal function (such as the logit and probit quantile functions) is commonly adopted to squash the acquired latent variable distribution, expressed as $\pi\left(x_{*}\right)=\sigma\left(f_{*}\right)[15]$. The class label is regarded as an independent variable and Bernoulli distribution. Therefore, the likelihood [18] is obtained for each target class:

$$
p(y \mid \mathbf{f})=\prod_{i=1}^{n} p\left(y_{i} \mid f_{i}\right)=\prod_{i=1}^{n} \sigma\left(y_{i} f_{i}\right)
$$

where $\mathbf{f}=\left\{f_{i} \mid i=1,2, \ldots, n\right\}, f_{i}=f\left(x_{i}\right)$. Assuming $f_{i}$ subject to GP distribution, the prior distribution of a latent function can be described [18]:

$$
p(\mathbf{f} \mid X)=\mathrm{N}(\mathbf{f} \mid \boldsymbol{\mu}, \mathbf{K})
$$

where $\boldsymbol{\mu}$ and $\mathbf{K}$ are mean vectors and positive covariance matrix, respectively.

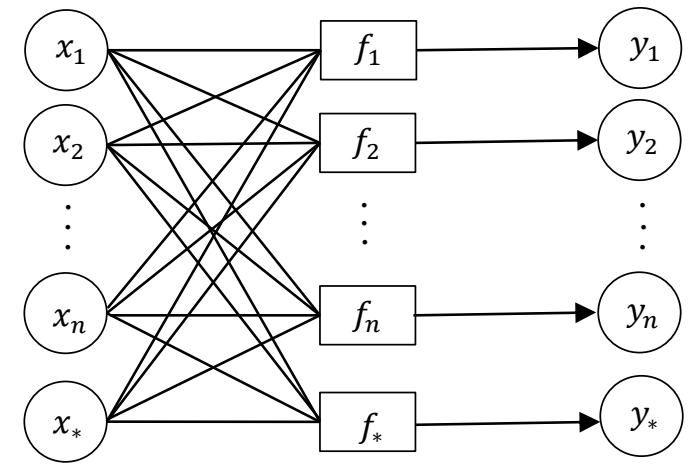

Figure 1. GPC classification procedure: $f$ represents the latent function, the left and right layer inputs are the data and output class labels, respectively. $y_{*}$ is the predicted class for test data $\mathbf{x}_{*}$. 
With a known GP prior to the latent function distribution, based on Bayes theorem, the posterior probability [18] can be deduced:

$$
\begin{aligned}
p(\mathbf{f} \mid y, X, \boldsymbol{\theta}) & =\frac{p(y \mid \mathbf{f}) p(\mathbf{f} \mid X)}{p(y \mid X, \boldsymbol{\theta})} \\
& =\frac{\mathrm{N}(\mathbf{f} \mid \mathbf{0}, \mathbf{K})}{p(y \mid X, \boldsymbol{\theta})} \prod_{i=1}^{n} \sigma\left(y_{i} f_{i}\right)
\end{aligned}
$$

where $\boldsymbol{\theta}$ represents the hyperparameter used for the parametrized GP prior. The marginal likelihood $p(y \mid X, \boldsymbol{\theta})=$ $\int p(y \mid \mathbf{f}) p(\mathbf{f} \mid X) d \mathbf{f}$. To develop a prediction for new test data $x_{*}$, we need to compute the probability distribution of latent function:

$$
p\left(f_{*} \mid x_{*}, y, X, \boldsymbol{\theta}\right)=\int p\left(f_{*} \mid \mathbf{f}, x_{*}, X, \boldsymbol{\theta}\right) p(\mathbf{f} \mid y, X, \boldsymbol{\theta}) d \mathbf{f}
$$

Thus, the class prediction probability for new test data $x_{*}$ is generated according to the formulation [18]:

$$
p\left(y_{*} \mid x_{*}, y, X, \boldsymbol{\theta}\right)=\int \sigma\left(y_{*} f_{*}\right) p\left(f_{*} \mid x_{*}, y, X, \boldsymbol{\theta}\right) d f_{*}
$$

However, the likelihood $p(y \mid \mathbf{f})$ in Equation (1) is a nonGaussian distribution so that the analytical solution of the marginal likelihood $p(y \mid X, \boldsymbol{\theta})$ and the latent function distribution in Equation (4) is unattainable: this is commonly resolved by Gaussian approximate inference or Markov chain Monte Carlo (MCMC) methods [18]. In this paper, Laplace approximation was applied to obtain a Gaussian distribution $q(\mathbf{f} \mid y, X, \boldsymbol{\theta})$ approximating with the posterior distribution $p(\mathbf{f} \mid y, X, \boldsymbol{\theta})$. The approximation value is obtained when the logposterior $\Psi(\mathbf{f})=\log p(\mathbf{f} \mid y, X, \boldsymbol{\theta})$ was expanded at the point of maximum posterior with a second-order Taylor expansion:

$$
p(\mathbf{f} \mid y, X, \boldsymbol{\theta}) \approx q(\mathbf{f} \mid y, X, \boldsymbol{\theta})=\mathrm{N}\left(\mathbf{f} \mid \widehat{\mathbf{f}},\left(\mathbf{K}^{-\mathbf{1}}+\mathbf{W}\right)^{-1}\right)
$$

where $\hat{\mathbf{f}}=\operatorname{argmax}_{\mathbf{f}} \Psi(\mathbf{f})$ and the negative Hessian $\mathbf{W}=$ $-\nabla \nabla \log p(y \mid \mathbf{f}) . \Psi(\mathbf{f})$ can be computed by the following equation according to Equation (3):

$$
\begin{aligned}
\Psi(\mathbf{f}) & =\log p(y \mid \mathbf{f})+\log p(\mathbf{f} \mid X) \\
& =\log p(y \mid \mathbf{f})-\frac{1}{2} \mathbf{f}^{\mathrm{T}} \mathbf{K}^{-\mathbf{1}} \mathbf{f}-\frac{1}{2} \log |\mathbf{K}|-\frac{\mathrm{n}}{2} \log (2 \pi)
\end{aligned}
$$

The approximation of the marginal likelihood $p(y \mid X, \boldsymbol{\theta})$ is deduced by the Taylor expansion:

$$
\begin{aligned}
\log p(y \mid X, \boldsymbol{\theta}) & =\log \int \exp (\Psi(\mathbf{f})) d \mathbf{f} \\
& =\log p(y \mid \hat{\mathbf{f}})-\frac{1}{2} \hat{\mathbf{f}}^{\mathrm{T}} \mathbf{K}^{-1} \hat{\mathbf{f}}-\frac{1}{2} \log |\mathbf{B}|
\end{aligned}
$$

in which $\mathbf{B}=\mathbf{K} \cdot\left(\mathbf{K}^{-\mathbf{1}}+\mathbf{W}\right)=\mathbf{I}+\mathbf{K W}$, the hyperparameter $\boldsymbol{\theta}$ is derived by maximizing the marginal likelihood $\log p(y \mid X, \boldsymbol{\theta})$ based on conjugate gradient method.

With the deduced Gaussian approximation of posterior, the mean and covariance of $f_{*}$ can be obtained. Therefore, the latent distribution term in Equation (4) for a test data $x_{*}$ can be described as Equation (9), and the class prediction probability is computed by Equation (10).

$$
\begin{aligned}
p\left(f_{*} \mid \mathbf{f}, x_{*}, X, \boldsymbol{\theta}\right) & =\mathrm{N}\left(\mathbf{k}_{*}^{\mathrm{T}} \mathbf{K}^{-1} \hat{\mathbf{f}}, \mathrm{k}_{* *}-\mathbf{k}_{*}^{\mathrm{T}}\left(\mathbf{K}+\mathbf{W}^{-\mathbf{1}}\right)^{-\mathbf{1}} \mathrm{k}_{*}\right) \\
\pi\left(f_{*}\right) & =\int \sigma\left(f_{*}\right) q\left(f_{*} \mid x_{*}, y, X, \boldsymbol{\theta}\right) d f_{*}
\end{aligned}
$$

\subsection{GAUSSIAN PROCESS MULTI-CLASS CLASSIFICATION}

Due to the wide variety of transformer faults, fault diagnoses using a Gaussian process classifier requires multi-class prediction. Multi-class classification is essentially an extension of a binary outcome, with more than two classes for recognition, which can be transformed into binary classification through one-versus-one (OVO) and one-versus-rest (OVR) approaches. OVR scheme is implemented by developing $C$ classifiers, where $C$ denotes the number of class. However, the predicted result of one sample may sometimes be outside the $C$ classifiers or subject to different classifiers, and all the classifiers would be retrained when adding a new classifier. In the OVR method, all training datasets are used as inputs to establish each binary classifier. Nevertheless, the OVO scheme only inputs the datasets with corresponding class label when training each binary classifier, which reduces the training time effectively. A

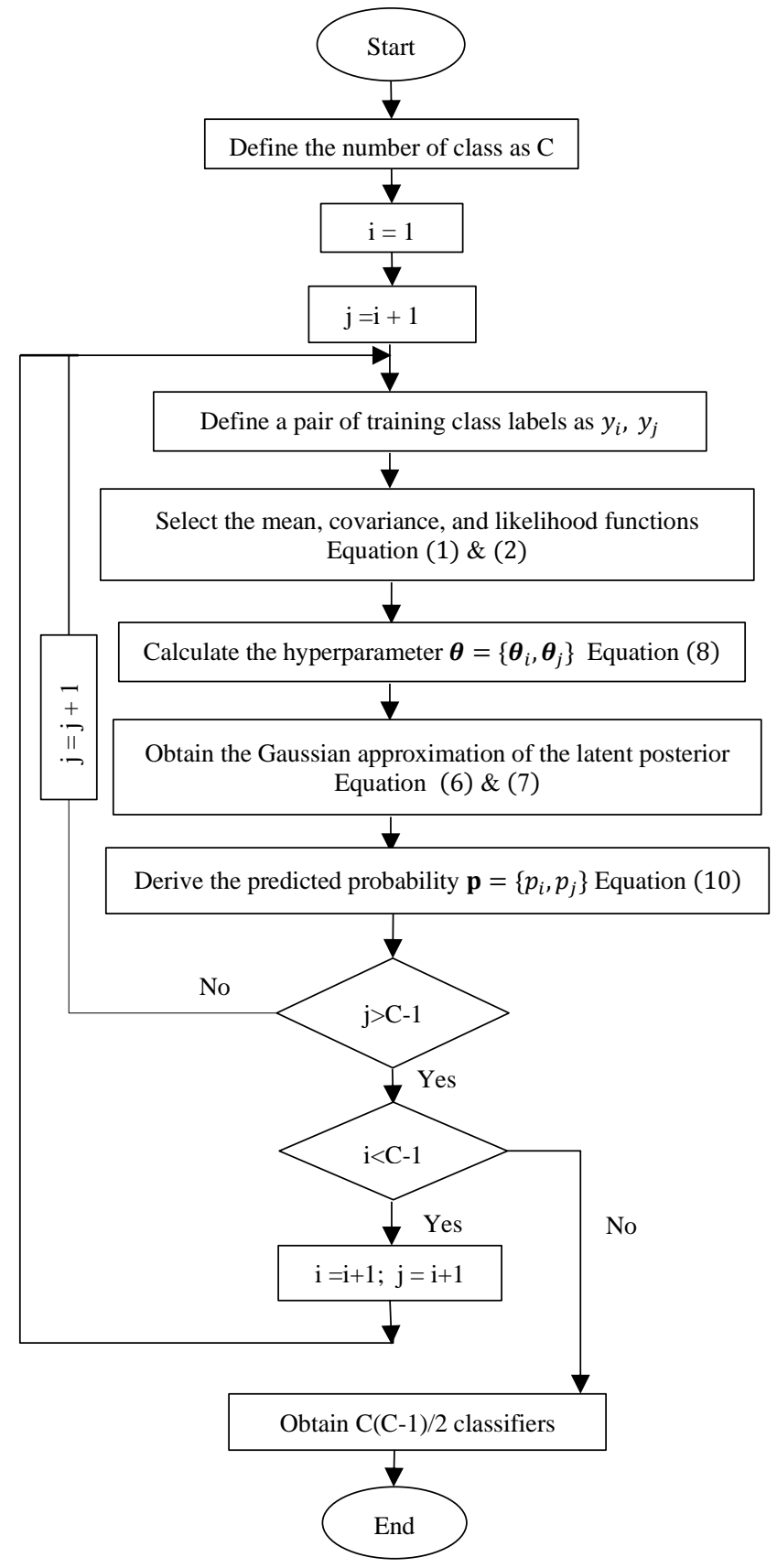

Figure 2. Process of training one versus one classifier 
voting scheme is applied to determine the prediction for new data which avoids ambiguous results. Therefore, the multi-class classification problem is converted into binary classification based on an OVO approach in this paper. The GPMC is realized by integrating several GP binary classifiers, and the final class label is determined by judging the maximum value of the output probability. The resolution steps are as follows:

1) Select a pair of classes from the training samples, denoted as $y_{i}$ and $y_{j}$, then $C(C-1) / 2$ Gaussian process binary classifiers are developed - the training procedure of one classifier is presented in Figure 2.

2) To classify a new test data $x_{*}$, all the classifiers trained in first step are employed, then determine the final predicted class $y_{*}$ by a voting scheme as: define a variable $\mathrm{n}_{k}(k=1,2, \ldots C)$ to count the number of times that each class appears as an output $\left(y_{n} \mid n=1,2, \ldots, C(C-1) / 2\right)$ in all classifiers. The initial value $\mathrm{n}_{k}=0$. The procedure for predicting the class label for $x_{*}$ is presented in Figure 3.

3) Input $x_{*}$ to the established classifiers. The hyperparameter vectors $\boldsymbol{\theta}=\left\{\boldsymbol{\theta}_{*}^{i}, \boldsymbol{\theta}_{*}^{j}\right\}$ in each classifier can be acquired based on Equation (8). The approximation $q(\mathbf{f} \mid y, X, \boldsymbol{\theta})$ of the latent posterior is calculated by Equation (6) and (7). With the known $q(\mathbf{f} \mid y, X, \boldsymbol{\theta})$, the probability distribution for latent function $q\left(f_{*} \mid x_{*}, y, X, \boldsymbol{\theta}\right)$ is obtained by combining Equation (4) and (9), and then the class prediction probability $(\mathbf{p}=$ $\left.\left\{p_{*}^{i}, p_{*}^{j}\right\}\right)$ can be computed by Equation (10).

4) For each classifier, find $\mathbf{p}_{\max }$ to ascertain the corresponding predicted class $k$, and $\mathrm{n}_{k}=\mathrm{n}_{k}+1$. The final class prediction for new data $x_{*}$ is determined by $\operatorname{Max}\left(\mathrm{n}_{k} \mid k=\right.$ $1,2, \ldots, C)$.

To assess the performance of the proposed GPMC method, other AI methods such as SVM, ANN, KNN, decision tree and logistic regression are used for comparative classification, discussed in Section 3.

\section{ARTIFICIAL INTELLIGENCE METHODS}

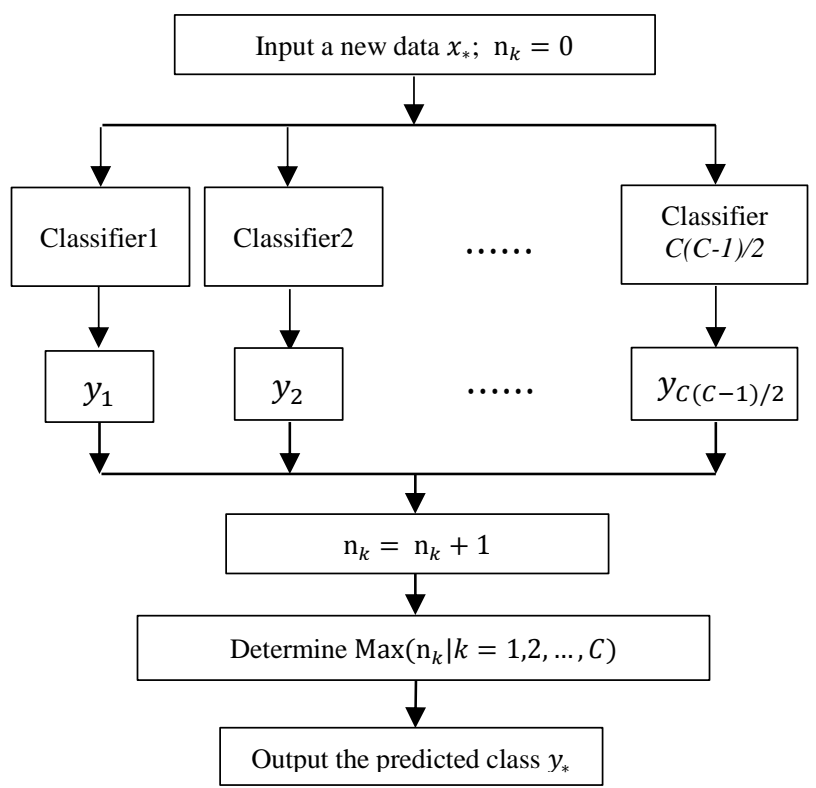

Figure 3. The procedure for predicting the class label for test data $\boldsymbol{x}_{*}$ : $\mathrm{n}_{k}(k=1,2, \ldots C)$ represents the number of times that each class appears as an output $y_{n}$.

\subsection{SUPPORT VECTOR MACHINE}

SVM is a kernel-based method, which has been wellemployed in regression and classification problems, which can suppress over-fitting due to structural risk minimization principles [19]. SVM for multi-classification is the expansion of a binary classification problem. It classifies different classes with a hyperplane. As for nonlinear classification, a kernel function $\boldsymbol{K}(x, y)$ is used to map the data from a low-dimension into a high-dimension and thus transfer the nonlinearity to linear separation. To determine the location of the decision plane, a margin needs to be defined which represents the minimum distance from the class point to the decision boundary and remains a maximum [20]. Given a set of training data $\mathrm{D}=$ $\left\{\left(x_{i}, y_{i}\right) \mid i=1,2, \ldots, \mathrm{n}\right\}$, The nonlinear SVM function can be expressed as:

$$
f(x)=\operatorname{sign}\left(\sum_{i=1}^{n} \alpha_{i} y_{i} \boldsymbol{K}\left(x, x_{i}\right)+b\right)
$$

where $\alpha_{i}$ are the Lagrange multipliers in the hyperplane and $\mathrm{b}$ denotes an offset. In this paper, a Gaussian kernel radial basis function (RBF) [21] is employed with the formation:

$$
\boldsymbol{K}(x, y)=\exp \left(-\frac{\|x-y\|^{2}}{2 \sigma^{2}}\right)
$$

The kernel parameter $\sigma$ controls the radial boundary of the function. An RBF is selected since: 1) it can realize the nonlinear mapping, and 2) there is less numerical complexity as a result of fewer parameters. Moreover, five-fold cross validation is used to adjust and optimize the model.

\subsection{ARTIFICIAL NEURAL NETWORK}

An ANN is a system which can realize complex logical operations while processing nonlinear relationships. ANNs can represent nonlinear systems without complicated mathematical models [22], and provides a layer-by-layer structure comprising a number of neurons and specific connection or rules between neurons. Each neuron represents an activation function: a weight is given to the connection between each two neurons. These model parameters are self-adapting according to the training data, which are tuned during each iteration by a convergent algorithm to obtain the minimum training error.

In this work, a multiple input multiple output (MIMO) ANN was developed, with the transformer key gases and fault classes as input and output, respectively. The ANN structure is

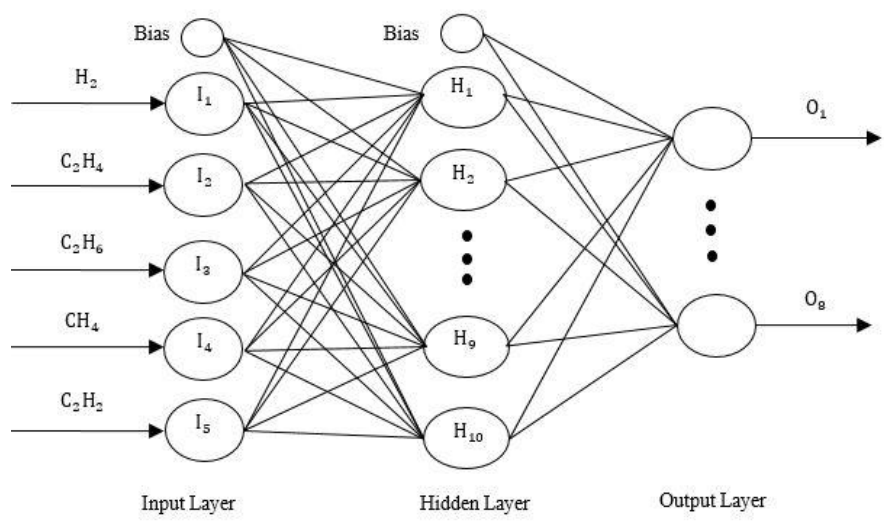

Figure 4. ANN topology 
described in Figure 4. A back-propagation learning algorithm was adopted in the ANN using the Levenberg-Marquardt algorithm to provide a local minimum value. The logisticsigmoid transfer function was employed in the hidden layer to add nonlinear factors and strengthen the performance of the ANN.

\subsection{K-NEAREST NEIGHBOUR}

$\mathrm{KNN}$ is an analogy-based algorithm used in various applications due its simple implementation and applications, such as data classification, character recognition and image identification. It is also known as a lazy learning algorithm, which stores all the training data instead of developing discriminative functions. The basic principle is to select $k$ training samples neighbouring the test data and determine the class prediction by majority-voting. The $k$ closest samples of examined point are chosen with the distance measurement [23]. The class label for the test data is predicted as the majority class assigned to the $k$ neighbouring samples.

\subsection{DECISION TREE}

The decision tree method uses a terse-tree structure to express the classification rule explicitly. Each internal node and leaf node represent the features and class label of training data, respectively. A root node is used to select the first branch for test data and split the data based on feature, then follow the branch to the next node until the data reaches the leaf node, after which a classification result is provided [24].

\subsection{LOGISTIC REGRESSION}

Logistic regression is a probabilistic model used for classification, which explains the relationship between features and output probability and is commonly applied for binomial classification [25]. Multinomial logistic regression model is used for multiclass problems, which is employed in this paper. The output is described as a probabilistic value by the sigmoidal function, mapping into the corresponding class.

\section{RESULT AND DISCUSSION}

\subsection{DGA DATA}

All methods applied in this paper are based on 7000 dissolved gas analysis (DGA) datasets containing both normal and abnormal states and as collected from several real oil-immersed transformers. In terms of identifying specific transformer states, quantities of dissolved gases reflect the level of oil decomposition which can reveal thermal or electrical faults. Therefore, the chosen datasets contain five key gas concentrations: $\mathrm{H}_{2}, \mathrm{C}_{2} \mathrm{H}_{4}, \mathrm{C}_{2} \mathrm{H}_{6}, \mathrm{CH}_{4}$, and $\mathrm{C}_{2} \mathrm{H}_{2}$, representing normal state and seven transformer fault types (denoted in Table 1) as follows: low-energy discharge, high-energy discharge, thermal fault of low temperature, thermal fault of medium temperature, thermal fault with high temperature, partial discharge and a combination of discharge and thermal faults [26]. The gas concentrations are used as inputs to the GPMC, SVM, ANN, KNN, decision tree and LR models, from which the outputs refer to predicted transformer health state (within defined classes).

The 7000 DGA datasets were divided into training data (4900 samples) and testing data (2100 samples) with a ratio of 7:3. The robustness of the data split is verified in Section 4.4. From Figure $5,1.8 \%$ of training data was D1; $3.6 \%$ was D2; $0.7 \%$ was DT; $0.2 \%$ was PD; $8.0 \%$ was T1; $17.4 \%$ was T2; $39.7 \%$ was T3; and $28.6 \%$ was NS. Training data with thermal faults $(65.1 \%)$ was much greater than those with discharge faults $(5.6 \%)$.

Table 1. Transformer health state definition

\begin{tabular}{cc}
\hline \hline Fault type & Fault indication \\
\hline D1 & Discharge of low-energy \\
D2 & Discharge of high-energy \\
DT & Combination of thermal fault and \\
discharges \\
T1 & Thermal fault, $\leqslant 300^{\circ} \mathrm{C}$ \\
T2 & Thermal fault, $>300^{\circ} \mathrm{C}, \leqslant 700^{\circ} \mathrm{C}$ \\
T3 & Thermal fault, $>700^{\circ} \mathrm{C}$ \\
PD & Partial discharges \\
NS & Normal state \\
\hline \hline
\end{tabular}

\subsection{MODEL COMPARISON}

The GPMC method can be regarded as an integration model of several one-versus-one binary classification models: hence, the predicted class with the maximum rating $\left(\mathrm{n}_{k}\right)$ is an output. In this paper, 28 Gaussian process binary classifiers were developed.

The MIMO-ANN has 5 inputs nodes in the input layer, 10 nodes in the hidden layer, and 8 nodes in the output layer, with 148 parameters including the weights and biases, the output was described in a binary vector related to the corresponding fault type: thus, the final predicted class was determined by the output value of 1 , such as $\mathrm{O}=\{1,0,0,0,0,0,0,0\}$ which indicates the predicted class is the first fault class D1. 4900 DGA samples shown in Figure 5 were used to train the model. This paper employed a squared inverse distance weighting method to select the neighbouring points and $k$ was set to 10 in the KNN models. The classification and regression tree (CART) algorithm was developed to predict the class label, which splits the node based on a Gini impurity. The split feature is chosen based on a greedy algorithm to minimize the cost function, and the final node was determined by the minimum number of training samples (predefined) of each node. A minimum costcomplexity pruning was applied in this work to overcome overfitting. Multinomial logistic regression model was implemented based on L2 regularization. The cost function was derived by maximum likelihood estimation and Newton's method was used to minimize the function; the maximum iteration was set to 100 .

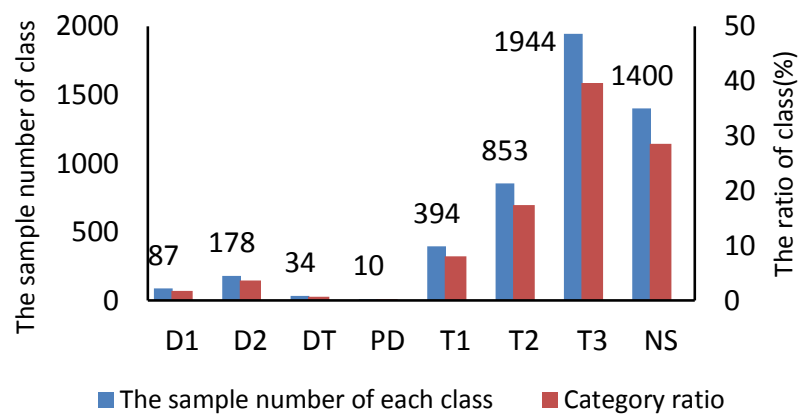

Figure 5. Training data distribution 
The performance of the methods was assessed by measuring the consistency of actual transformer health condition against predicted classification. Table 2 illustrates the classification accuracy for all six models: GPMC, SVM, ANN, KNN, decision tree and LR. The accuracy distribution among all classes is clearly tabulated.

1) Thermal faults

Thermal faults included T1, T2 and T3. The proposed GPMC diagnosed high-temperature faults with the highest accuracy $(99.9 \%)$, which indicates that all the training fault datasets for T3 (and the normal DGA datasets) were almost classified correctly. In addition, $99.2 \%$ and $99.1 \%$ of samples with $\mathrm{T} 1$ and $\mathrm{T} 2$, respectively were exactly predicted. As for T3 prediction, the KNN and decision tree models rendered a lower accuracy in comparison to the GPMC model, with $98.3 \%$ and $97.5 \%$, respectively. The LR model misclassified T2 with a $19 \%$ difference, higher than the other models. With an accuracy of $80.2 \%$, recognition of T1 in the ANN model was lower than the other models. It can therefore be concluded that the GPMC model delivered the best performance in all of the thermal fault classification evaluations.

2) Electrical faults

D1, D2 and PD represented electrical faults. The GPMC, SVM, ANN, KNN, decision tree and LR models achieved $99.4 \%, 96.1 \%, 96.6 \%, 87.1 \%, 84.8 \%$ and $97.8 \%$ accuracy, respectively for prediction of the D2 fault. All samples for D1 and PD classification were diagnosed precisely by the GPMC model with $100 \%$ accuracy. However, the SVM and ANN models misclassified all PD datasets, while the KNN, decision tree and LR models yielded a poor performance with $20.0 \%$, $40.0 \%$ and $40.0 \%$ accuracy, respectively for prediction of the PD fault type. The ANN model showed the lowest accuracy $(59.8 \%)$ for discrimination of D1.

3) Multiple faults \& Normal state

Power transformer faults can arise from the impact of both thermal and electrical stresses. The occurrence of multiple fault classes in this evaluation is denoted as DT. The GPMC model delivered a highly accurate classification (91.2\%), followed by $79.4 \%$ accuracy from the decision tree model. The SVM and LR models diagnosed DT with only $38.2 \%$ and $11.8 \%$ accuracy, respectively, and the ANN model classified DT incorrectly.

99.9\% accuracy was attained by the GPMC model for NS classification. The SVM and ANN models rendered accuracies of $98.6 \%$ and $98.4 \%$, respectively, while the LR model gave the lowest comparative performance $(85.7 \%)$ in this instance.

An accuracy index was introduced to elaborate the performance evaluation of the models, derived as the ratio of number of correct predictions over the total number of fault datasets. Overall diagnosis accuracy obtained was: $99.6 \%$, $94.3 \%, 94.1 \%, 95.0 \%, 94.4 \%$ and $89.1 \%$ for the GPMC, SVM, ANN, KNN, decision tree and LR models, respectively. The GPMC model only misclassified $0.4 \%$ of 4900 datasets, yielding a smaller error between predicted class and true transformer health condition compared to the other models.

Table 2. Classification accuracy of six diagnostic models (\%)

\begin{tabular}{|c|c|c|c|c|c|c|c|c|c|}
\hline & $\begin{array}{c}\text { Predicted } \\
\text { class }\end{array}$ & D1 & D2 & DT & $\begin{array}{c}\text { True class } \\
\text { PD }\end{array}$ & $\mathrm{T} 1$ & $\mathrm{~T} 2$ & T3 & NS \\
\hline \multirow{8}{*}{ GPMC } & D1 & 100 & 0.6 & 0 & 0 & 0 & 0 & 0 & 0 \\
\hline & D2 & 0 & 99.4 & 0 & 0 & 0 & 0 & 0 & 0 \\
\hline & DT & 0 & 0 & 91.2 & 0 & 0 & 0.1 & 0 & 0 \\
\hline & PD & 0 & 0 & 0 & 100 & 0 & 0 & 0 & 0 \\
\hline & $\mathrm{T} 1$ & 0 & 0 & 0 & 0 & 99.2 & 0.1 & 0 & 0 \\
\hline & $\mathrm{T} 2$ & 0 & 0 & 5.9 & 0 & 0.5 & 99.1 & 0.1 & 0.07 \\
\hline & $\mathrm{T} 3$ & 0 & 0 & 2.9 & 0 & 0 & 0.35 & 99.9 & 0.07 \\
\hline & NS & 0 & 0 & 0 & 0 & 0.3 & 0.35 & 0 & 99.9 \\
\hline \multirow[t]{3}{*}{ Accuracy } & \multicolumn{9}{|c|}{$99.6 \%$} \\
\hline & Predicted & & & & True class & & & & \\
\hline & class & D1 & D2 & DT & PD & $\mathrm{T} 1$ & $\mathrm{~T} 2$ & $\mathrm{~T} 3$ & NS \\
\hline \multirow{8}{*}{ SVM } & D1 & 88.5 & 2.8 & 0 & 0 & 0 & 0 & 0 & 0 \\
\hline & D2 & 8.1 & 96.1 & 0 & 0 & 0 & 0 & 0 & 0 \\
\hline & DT & 0 & 0 & 38.2 & 0 & 0 & 0.1 & 0 & 0.1 \\
\hline & PD & 0 & 0 & 0 & $\mathbf{0}$ & 0 & 0 & 0 & 0 \\
\hline & T1 & 0 & 0 & 0 & 30 & 93.4 & 0.1 & 0 & 0.1 \\
\hline & $\mathrm{T} 2$ & 0 & 0 & 2.9 & 0 & 3.0 & 94 & 1.1 & 1.2 \\
\hline & $\mathrm{T} 3$ & 0 & 0 & 0 & 0 & 0 & 1.2 & 93.2 & 0 \\
\hline & NS & 3.4 & 1.1 & 58.9 & 70 & 3.6 & 4.6 & 5.7 & 98.6 \\
\hline Accuracy & \multicolumn{9}{|c|}{$94.3 \%$} \\
\hline & Predicted & & & & True class & & & & \\
\hline & class & D1 & D2 & DT & PD & $\mathrm{T} 1$ & $\mathrm{~T} 2$ & $\mathrm{~T} 3$ & NS \\
\hline \multirow{8}{*}{ ANN } & D1 & 59.8 & 2.2 & 0 & 0 & 0 & 0 & 0 & 0 \\
\hline & D2 & 39.1 & 96.6 & 2.9 & 0 & 0 & 0 & 0 & 0 \\
\hline & DT & 0 & 0 & 0 & 0 & 0 & 0 & 0 & 0 \\
\hline & PD & 0 & 0 & 0 & 0 & 0 & 0 & 0 & 0 \\
\hline & $\mathrm{T} 1$ & 0 & 0 & 0 & 50 & 80.2 & 0.1 & 0 & 0.3 \\
\hline & $\mathrm{T} 2$ & 0 & 0.6 & 5.9 & 10 & 15.5 & 95.9 & 2.3 & 0.4 \\
\hline & T3 & 0 & 0 & 11.8 & 0 & 0 & 0 & 96.6 & 0.9 \\
\hline & NS & 1.1 & 0.6 & 79.4 & 40 & 4.3 & 4 & 1.1 & 98.4 \\
\hline Accuracy & \multicolumn{9}{|c|}{$94.1 \%$} \\
\hline
\end{tabular}




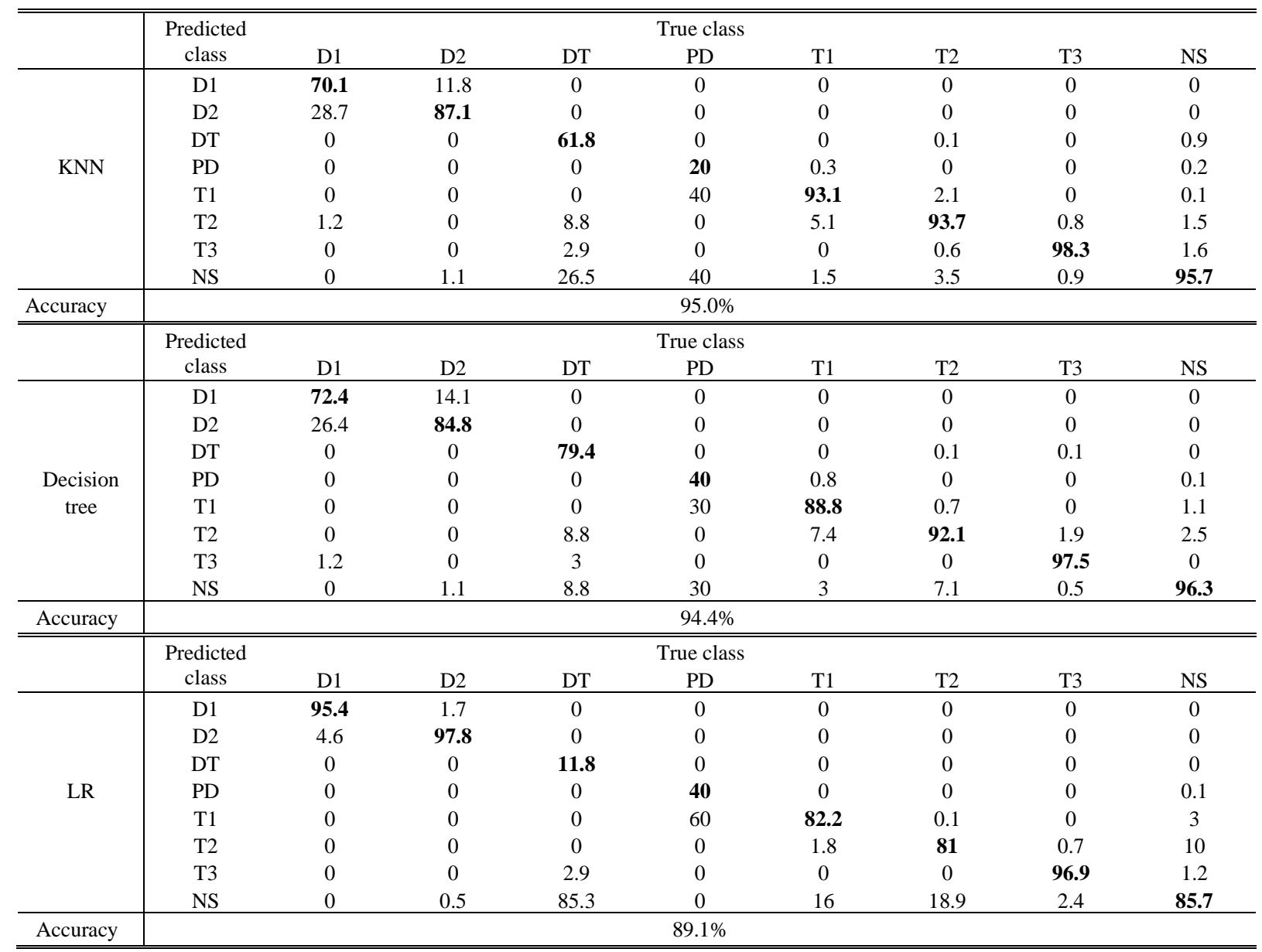

As shown in Figure 5, it is apparent that the volume of training samples for D1, D2, DT and PD fault was only 1.8\%, 3.2\%, $0.7 \%$ and $0.2 \%$ (87 samples, 178 samples, 34 samples, 10 samples) in the training datasets (which was much less than those available for the other fault types), and can be regarded as small-scale datasets. The training accuracy of small-scale datasets in the six AI models are tabulated in Table 3, which presents that the highest prediction accuracy of D1, D2, DT and DT are obtained by GPMC, with $100 \%, 99.4 \%, 91.2 \%$ and $100 \%$ accuracy, respectively. GPMC performs with high and consistent accuracy for DT and PD classification compared with the other models. Therefore, it can be concluded that the proposed GPMC model performed best among the comparative models in all fault type classifications, particularly for the prediction of small-scale datasets - this affirms that the GPMC model is capable of diagnosing fault types with comparatively small errors, despite access to limited training samples.

Table 3. Classification accuracy of small-scale datasets (\%)

\begin{tabular}{ccccccc}
\hline \hline Accuracy(\%) & GPMC & SVM & ANN & KNN & $\begin{array}{c}\text { Decision } \\
\text { tree }\end{array}$ & LR \\
\hline D1 & $\mathbf{1 0 0}$ & 88.5 & 59.8 & 70.1 & 72.4 & 95.4 \\
D2 & $\mathbf{9 9 . 4}$ & 96.1 & 96.6 & 87.1 & 84.8 & 97.8 \\
DT & $\mathbf{9 1 . 2}$ & 38.2 & 0 & 61.8 & 79.4 & 11.8 \\
PD & $\mathbf{1 0 0}$ & 0 & 0 & 20.0 & 40 & 40.0 \\
\hline \hline
\end{tabular}

A computational complexity comparison of the AI methods evaluated is presented in Table 4, where $n$ and $C$ are the number of training samples and class, respectively; $k$ denotes the number of neighbours; $d$ is the dimension of each data sample and $t$ is the number of epochs. It can be found that the highest time complexity is described by GPMC $\left(\mathrm{O}\left((2 n / C)^{3}\right) \approx\right.$ $\left.\mathrm{O}(n)^{3}\right)$, which is followed by SVM $\left(\mathrm{O}\left(n^{2}\right)\right)$, and KNN has the lowest time complexity $(\mathrm{O}(k n d) \approx \mathrm{O}(n))$. It indicates that GPMC takes more time to train the model than other models.

Table 4. Time complexity comparison

\begin{tabular}{cc}
\hline \hline Model & Time complexity \\
\hline GPMC & $O\left((2 n / C)^{3}\right)$ \\
SVM & $O\left(n^{2}\right)$ \\
ANN & $O(148 n t)$ \\
KNN & $O(k n d)$ \\
Decision tree & $O(n \log (n) d)$ \\
LR & $O((d+1) C n)$ \\
\hline \hline
\end{tabular}

\subsection{CASE STUDY WITH AI METHODS}

2100 testing data samples were used to verify model accuracy and robustness. In addition to the GPMC, SVM, ANN, KNN, decision tree and LR models, conventional DGA interpretations (Dornenburg ratio, IEC 60599, key gases) were used for fault prediction, as presented in Section 4.5.

Distribution of the eight condition types in this case study are presented in Table 5 as follows: 35 cases (with D1); 86 cases (with D2); 20 cases (with DT); 4 cases (with PD); 174 cases (with T1); 366 cases (with T2); 815 cases (with T3); and 600 cases (with NS). The table also reveals a confusion matrix for intuitive fault misclassification in the GPMC model: thus, 5 cases (with D1) were misclassified (as D2); 5 cases (of D2) 
were assigned incorrectly (as D1). The model diagnosed 13 of the 20 DT datasets correctly. In PD prediction, 3 cases were classified wrongly (D1, T1 and NS) and there were 11 datasets with $\mathrm{T} 1$ predicted as T2 (and a further 3 datasets with T1 predicted as NS). The GPMC model predicted 347 cases of T2 exactly, whereas for T3, the model misclassified 3 cases (as D1), a further 3 cases (as T2) and additional 9 cases (as NS); however, $95.7 \%$ of the NS datasets were correctly predicted.

Table 5. Confusion matrix for GPMC model

\begin{tabular}{c|ccccccccc}
\hline \hline & Predicted & \multicolumn{8}{c}{ True class } \\
& class & D1 & D2 & DT & PD & T1 & T2 & T3 & NS \\
\hline \multirow{5}{*}{ GPMC } & D1 & $\mathbf{3 0}$ & 5 & 2 & 1 & 2 & 0 & 3 & 4 \\
& D2 & 5 & $\mathbf{8 1}$ & 0 & 0 & 0 & 0 & 0 & 0 \\
& DT & 0 & 0 & $\mathbf{1 3}$ & 0 & 0 & 2 & 0 & 0 \\
& PD & 0 & 0 & 0 & $\mathbf{1}$ & 0 & 0 & 0 & 0 \\
& T1 & 0 & 0 & 0 & 1 & $\mathbf{1 5 8}$ & 4 & 0 & 5 \\
& T2 & 0 & 0 & 4 & 0 & 11 & $\mathbf{3 4 7}$ & 3 & 13 \\
& T3 & 0 & 0 & 1 & 0 & 0 & 1 & $\mathbf{8 0 0}$ & 4 \\
& NS & 0 & 0 & 0 & 1 & 3 & 12 & 9 & $\mathbf{5 7 4}$ \\
\hline & Total cases & 35 & 86 & 20 & 4 & 174 & 366 & 815 & 600 \\
\hline \hline
\end{tabular}

An accuracy comparison for all the AI models used in this study (GPMC, SVM, ANN, KNN, decision tree, and LR) is tabulated in Table 6.

The SVM and LR performed with the highest accuracy, $91.4 \%$ and $98.8 \%$ for D1 and D2 diagnoses, respectively (with $5.7 \%$ and $4.6 \%$ greater accuracy compared to the GPMC model). $95 \%$ accuracy for DT prediction was attained by the decision tree model (higher than the comparative models), followed by accuracies of $70.0 \%$ and $65.0 \%$ for the SVM and GPMC models, respectively. The KNN model classified 94.3\% of T1 cases correctly, while GPMC and ANN models rendered $90.8 \%$ and $76.4 \%$ accuracies, respectively. Prediction of T2 (with the SVM model) was $95.1 \%$ accurate, while an accuracy of $98.2 \%$ was achieved using the GPMC model for T3 classification. The best prediction accuracy for the PD faults was $25.0 \%$ rendered by the GPMC, KNN, decision tree, and LR model, but the ANN model for DT and PD classification was unsatisfactory: insufficient training samples (for PD and DT) may have affected the ANN prediction - a significant quantity of training data is essential to guarantee good prediction precision. In addition, it can be observed that excluding PD from the study (which tends to have only a few observations) SVM performs mostly better than GPMC and other diagnostic models, which means the performance of SVM is comparable with the proposed GPMC. However, it can be determined that the accuracy of SVM is slightly better than GPMC for some classes prediction, and the accuracy difference between the two models is significant when GPMC has better classification accuracy (especially for PD prediction). PD faults are not common in practice, but can cause severe damage to transformers: the results obtained for prediction of PD faults in this work is therefore particularly significant. Although the performance of GPMC is not ideal in PD classification (mainly due to the imbalanced training datasets), it is better than SVM (with 0\%). Therefore, diagnostic accuracy for each condition classified by the GPMC model was more stable, compared to the other models.

As shown in Table 6, the GPMC model accuracy was $95.4 \%$, slightly exceeding SVM and KNN model outcomes of $94.3 \%$ and $94.2 \%$, respectively. The LR model has the lowest accuracy with $88.0 \%$. Accordingly, and as demonstrated in the evaluation in this paper, diagnostic assessment of transformer faults using the GPMC model tends to more precise than the other AI models used in this work.

Table 6. Accuracy comparison for five diagnostic models (\%)

\begin{tabular}{ccccccc}
\hline \hline $\begin{array}{c}\text { Fault } \\
\text { Type }\end{array}$ & GPMC & SVM & ANN & KNN & $\begin{array}{c}\text { Decision } \\
\text { tree }\end{array}$ & LR \\
\hline D1 & 85.7 & 91.4 & 74.3 & 71.4 & 80.0 & 88.6 \\
D2 & 94.2 & 97.6 & 90.7 & 82.6 & 95.3 & 98.8 \\
DT & 65.0 & 70.0 & 0 & 60.0 & 95.0 & 0 \\
PD & 25.0 & 0 & 0 & 25.0 & 25.0 & 25.0 \\
T1 & 90.8 & 92.0 & 76.4 & 94.3 & 83.3 & 79.3 \\
T2 & 94.8 & 95.1 & 94.8 & 94.0 & 89.9 & 81.4 \\
T3 & 98.2 & 92.1 & 95.0 & 97.5 & 96.7 & 95.3 \\
NS & 95.7 & 98.6 & 96.7 & 94.3 & 96.0 & 86.5 \\
\hline Overall & 95.4 & 94.3 & 92.3 & 94.2 & 93.7 & 88.0 \\
\hline \hline
\end{tabular}

\subsection{ROBUSTNESS OF DATA SPLIT}

To verify the robustness of the results with respect to the split used in this paper in terms of training and test data, the training and test accuracy of six AI models (based on different split ratios) are presented as below. 7000 datasets were divided into training data and test data according to the ratio of 6:4 (4200 samples: 2800 samples), 7:3 (4900 samples: 2100 samples) and 8:2 (5600 samples: 1400 samples), respectively. From Table 7 $\& 8$, it is apparent that the training accuracy of the five AI models (excluding LR model) based on the ratio of 7:3 is better than the other ratios. The test accuracy of the models with the ratio of $6: 4$ is the lowest. When the data was divided by the ratio of 7:3, the test accuracy of the KNN and decision tree are better than the models with the ratio of $8: 2$. SVM and ANN have slightly lower accuracy, and GPMC performs with the same precision. For the LR model based on the ratio of 7:3, although the training accuracy is lower than other ratios, the test accuracy is the highest. Therefore, it can be concluded that the split used in the paper $(7: 3)$ would be more robust, compared to other splitting.

Table 7. Training accuracy using different split ratio of data $(\%)$

\begin{tabular}{c|cccccc}
\hline \hline \multirow{2}{*}{$\begin{array}{l}\text { Split } \\
\text { ratio }\end{array}$} & GPMC & SVM & ANN & KNN & $\begin{array}{c}\text { Decision } \\
\text { tree }\end{array}$ & LR \\
\cline { 2 - 7 } & & & & & 93.8 & 89.6 \\
$6: 4$ & 99.6 & 94.0 & 91.7 & 93.7 & 94.4 & 89.1 \\
$7: 3$ & 99.6 & 94.3 & 94.5 & 95.0 & 93.0 & 89.4 \\
$8: 2$ & 99.4 & 94.1 & 93.8 & 94.2 & 93.0 \\
\hline \hline
\end{tabular}

\begin{tabular}{c|cccccc}
\multicolumn{7}{c}{ Table 8. Test accuracy using different split ratio of data (\%) } \\
\hline \hline \multirow{2}{*}{$\begin{array}{c}\text { Split } \\
\text { ratio }\end{array}$} & \multicolumn{5}{c}{ Classification model } \\
\cline { 2 - 7 } & GPMC & SVM & ANN & KNN & $\begin{array}{c}\text { Decision } \\
\text { tree }\end{array}$ & LR \\
\hline $6: 4$ & 95.2 & 93.7 & 91.7 & 94.1 & 91.9 & 85.0 \\
$7: 3$ & 95.4 & 94.3 & 93.6 & 94.2 & 93.7 & 88.0 \\
$8: 2$ & 95.4 & 94.9 & 93.7 & 91.7 & 92.6 & 87.3 \\
\hline \hline
\end{tabular}

\subsection{COMPARISON OF AI METHODS AND CONVENTIONAL METHODS}

Consistency and accuracy are introduced to compare the performance of conventional methods with artificial approaches [27], and these are calculated based on the total successful predictions in each method as follows: 


$$
\begin{gathered}
S_{F n}=\frac{P_{F n}}{\text { Number of cases with Fn fault }} \times 100 \\
\text { Consistency }=\frac{\sum_{1}^{F n} S_{F n}}{\text { Number of fault types }} \\
\text { Accuracy }=\frac{\sum_{1}^{F n} P_{F n}}{\text { Total cases }-" \text { No result "cases }}
\end{gathered}
$$

Where $S_{F n}$ shows the percentage of correct prediction for fault type $F n$, and $P_{F n}$ is the number of correct diagnoses in Equation (13). Due to boundary limitations in conventional methods, several cases were undefined, which is represented by the "No

\begin{tabular}{|c|c|c|c|}
\hline Percentage $(\%)$ & $\begin{array}{c}\text { Consistency } \\
\text { (C) }\end{array}$ & $\begin{array}{c}\text { Accuracy } \\
\text { (A) }\end{array}$ & Average of $\mathrm{C}$ and $\mathrm{A}$ \\
\hline Dornenburg ratio & 37.9 & 79.0 & 58.5 \\
\hline IEC 60599 & 43.6 & 42.4 & 43.0 \\
\hline Key gases & 68.6 & 37.8 & 53.2 \\
\hline SVM & 79.6 & 94.3 & 87.0 \\
\hline ANN & 66.0 & 92.3 & 79.1 \\
\hline KNN & 77.4 & 94.2 & 85.8 \\
\hline Decision tree & 82.7 & 93.7 & 88.2 \\
\hline LR & 69.4 & 88.0 & 78.7 \\
\hline GPMC & 81.2 & 95.4 & 88.3 \\
\hline
\end{tabular}
result" cases in Equation (15).

Table 9. Consistency and Accuracy of all methods

Table 9 shows the consistency, accuracy, and average value in the nine chosen methods presented: the accuracy of the AI models is generally better than the cited conventional methods. Use of the Dornenburg ratio method has a higher accuracy than IEC 60599 and the key gas method, but consistency is limited, since the ratio in some cases is out-of-range. The most consistent method (among conventional approaches) is the key gas method, which is superior to outcomes from the ANN model. In terms of all approaches discussed, the decision tree method is the most consistent, immediately followed by the GPMC model. The highest accuracy is yielded by GPMC model. It can therefore be concluded that the GPMC model delivers the best overall performance since the average accuracy exceeds that determined by conventional methods and the other AI models.

\section{CONCLUSION}

In this paper, a GPMC model approach is proposed based on a Gaussian process binary classifier to render multi-class classification for applications in incipient power transformer faults. The common AI models, SVM, ANN, KNN, decision tree and LR provide a fair and appropriate basis for GPMC model comparison. DGA conventional methods are employed in further assessment alongside all AI models. Large-scale DGA datasets were used for model implementation: dissolved (five-key) gas concentrations were viewed as feature inputs to determine the prediction accuracy of transformer health condition. Experimental evaluation and results have indicated that AI models yield better performance compared to conventional methods, and the proposed GPMC model renders $>95 \%$ accuracy - in most instances better than the other AI models. The two prime benefits of the GPMC model are: 1) the GPMC model performs better than other models when smallscale datasets (with D1, D2, DT and PD type) are used (the other models require high volumes of training data to learn fault characteristics in order to achieve a good performance); and 2) prediction results from the GPMC model are interpretable due to the probabilistic outputs obtained with this approach.

In the GPMC model, case study prediction accuracy was lower than the model training accuracy (by 4.2\%). It can also be deduced that unbalanced training data might influence the effectiveness of diagnostic models. Therefore, future work will focus on: 1) forming solutions with limited data for the PD and DT classes; and 2) use of different kernel functions while assessing the impact of data noise for generalization of the GPMC model. This work forms part of ongoing research in the assessment of AI methods for transformer diagnostics which are essential in establishing and delivering accuracy, consistency and diversity in power system key asset management and shaping strategic policies for preemptive maintenance and replacement.

\section{ACKNOWLEDGMENT}

This work was supported by the Engineering and Physical Sciences Research Council. The authors would like to express their gratitude to General Electric for collecting and providing DGA data used in this paper.

\section{REFERENCES}

[1] A. Abu-Siada and S. Islam, "A new approach to identify power transformer criticality and asset management decision based on dissolved gas-in-oil analysis," IEEE Trans. Dielectr. Electr. Insul., vol. 19, no. 3, pp. 1007-1012, June 2012.

[2] N. A. Bakar, A. Abu-Siada, and S. Islam, "A review of dissolved gas analysis measurement and interpretation techniques," IEEE Electr. Insul. Mag., vol. 30, no. 3, pp. 39-49, May-June 2014.

[3] IEEE Guide for the Interpretation of Gases Generated in Mineral OilImmersed Transformers, IEEE Standard C57.104-2019, 2019-11-1.

[4] T. Kari et al, "An integrated method of ANFIS and Dempster-Shafer theory for fault diagnosis of power transformer," IEEE Trans. Dielectr. Electr. Insul., vol. 25, no. 1, pp. 360-371, Feb. 2018.

[5] C. Guo et al, "Transformer failure diagnosis using fuzzy association rule mining combined with case-based reasoning," IET Gener. Transm. Distrib., vol. 14, no. 11, pp. 2202 - 2208, June 2020.

[6] Y. Benmahamed, M. Teguar, and A. Boubakeur, "Application of SVM and KNN to Duval Pentagon 1 Transformer Oil Diagnosis," IEEE Trans. Dielectr. Electr. Insul., vol. 24, no. 6, pp. 3443-3451, Dec. 2017.

[7] C. Xiang et al, "Graphic approaches for faults diagnosis for Camellia insulating liquid filled transformers based on dissolved gas analysis," IEEE Trans. Dielectr. Electr. Insul., vol. 25, no. 5, pp. 1897-1903, Oct. 2018.

[8] K. Benhamed et al, "Feature selection for effective health index diagnoses of power transformers," IEEE Trans. Power Deliv., vol. 33, no. 6, pp. 3223-3226, Dec. 2018.

[9] J. Jiang et al, "Dynamic fault prediction of power transformers based on hidden Markov model of dissolved gases analysis," IEEE Trans. Power Deliv., vol. 34, no. 4, pp. 1393-1400, Aug. 2019.

[10] J. I. Aizpurua et al, "Power transformer dissolved gas analysis through Bayesian networks and hypothesis testing," IEEE Trans. Dielectr. Electr. Insul., vol. 25, no. 2, pp. 494-506, Apr. 2018.

[11] M. Yang and L. Hu, "Intelligent fault types diagnostic system for dissolved gas analysis of oil-immersed power transformer," IEEE Trans. Dielectr. Electr. Insul., vol. 20, no. 6, pp. 2317-2324, Dec. 2013.

[12] J. Dai et al, "Dissolved gas analysis of insulating oil for power transformer fault diagnosis with deep belief network," IEEE Trans. Dielectr. Electr. Insul., vol. 24, no. 5, pp. 2828-2835, Oct. 2017.

[13] L. Wang, T. Littler, and X. Liu, "Artificial intelligence model for transformer fault diagnosis using a constructed database," presented at the Conference on AC and DC Power Transmission, Xi'an, China, July 2020.

[14] R. M. Neal, "Bayesian learning for neural networks," PhD dissertation, Dept. of Comput. Science, University of Toronto, 1995. 
[15] C.E. Rasmussen and C.K.I. Williams, Gaussian Processes for Machine Learning, the MIT Press, 2006, pp. 33-77.

[16] C.K.I. Williams and C.E. Rasmussen, "Gaussian processes for regressions, " in Advances in Neural Information Processing System, vol. 8, D. Touretzky, M. Mozer and M. Hasselmo, Ed. Cambridge MA, 1996, pp. 514-520.

[17] H.-C. Kim and Z. Ghahramani, "Bayesian Gaussian process classification with the EM-EP algorithm," IEEE Trans. Pattern Anal. Mach. Intell., vol. 28, no. 12, pp. 1948-1959, Dec. 2006.

[18] H. Nickisch and C. Rasmussen, "Approximations for binary Gaussian process classification," J. Mach. Learn. Res., vol. 9, pp. 2035-2078, 2008.

[19] S. Xiong, H. Liu, and X. Niu, "Fuzzy support vector machines based on FCM clustering, " Int. conf. Mach. Learn. Cybern. (ICMLC), 2005, pp. 2608-2613.

[20] K. Muller et al, "An introduction to kernel-based learning algorithms," IEEE Trans. Neural Netw., vol. 12, no. 2, pp. 181-201, Mar. 2001.

[21] M.A. Hearst et al, "Support Vector Machines," IEEE Intell. Syst. Appl., vol. 13, no. 4, pp. 18-28, 1998.

[22] Z. Wang, Y. Liu, and P. Griffin, "Neural Net and Expert System Diagnose Transformer Faults," IEEE Comput. Appl. Power, vol. 13, no.1, pp. 5055, Jan. 2000.

[23] A. Shintemirov, W. Tang, and Q. Wu, "Power transformer fault classification based on dissolved gas analysis by implementing bootstrap and genetic programming," IEEE Trans. Syst. Man Cybern. C Appl. Rev., vol. 39, no. 1, pp. 69-79, Jan. 2009.

[24] J. R. Quinlan, "Decision trees and decision making," IEEE Trans. Syst. Man Cybern., vol. 20, no. 2, pp. 339-346, Mar./Apr. 1990.

[25] M. Yin et al, "Robust multinomial logistic regression based on RPCA," IEEE J. Sel. Topics Signal Process., vol. 12, no. 6, pp. 1144-1154, Dec. 2018.

[26] J. Faiz and M. Soleimani, "Dissolved gas analysis evaluation in electric power transformers using conventional methods a review," IEEE Trans Dielectr. Electr. Insul., vol. 24, no. 2, pp. 1239-1248, Apr. 2017.

[27] J. Faiz and M. Soleimani, "Assessment of computational intelligence and conventional dissolved gas analysis methods for transformer fault diagnosis," IEEE Trans. Dielectr. Electr. Insul., vol. 25, no. 5, pp. 17981806, Oct. 2018. 